\title{
Hamiltonian formalism for nonlocal Lagrangians
}

\author{
J. Llosa and J. Vives \\ Departament de Física Fonamental, Universitat de Barcelona Diagonal, 647; \\ E-0828 - Barcelona, Spain
}

(Received 30 June 1993; accepted for publication 25 January 1994)

\begin{abstract}
A Hamiltonian formalism is set up for nonlocal Lagrangian systems. The method is based on obtaining an equivalent singular first order Lagrangian, which is processed according to the standard Legendre transformation and then, the resulting Hamiltonian formalism is pulled back onto the phase space defined by the corresponding constraints. Finally, the standard results for local Lagrangians of any order are obtained as a particular case.
\end{abstract}

\section{INTRODUCTION}

Nonlocal interactions have been proposed several times in theoretical physics with a variety of purposes. A nonexhaustive examination of the literature reveals their occurrence in topics as diverse as: Fokker-Wheeler-Feynman electrodynamics, ${ }^{1}$ regularized local field theories, ${ }^{2}$ some models of meson-nucleon interaction ${ }^{3}$ and, very recently, string field theory, ${ }^{4,5}$ and semiclassical gravity. $^{6}$

However, many of these attempts were abandoned in the past due to the lack of a Hamiltonian formalism for nonlocal Lagrangian systems, which seems a necessary intermediate step towards quantization. Indeed, there is nothing similar to the Legendre transformation for nonlocal Lagrangians. Even the phase space itself is not well defined in the nonlocal case.

Regular first order Lagrangians lead to a set of Euler-Lagrange equations that can be solved to write the generalized accelerations $\ddot{q}$ in terms of the generalized coordinates $q$ and velocities $\dot{q}$ and time $t$. Then, the existence and uniqueness theorems for ordinary differential systems ${ }^{7}$ state that for any given initial point $\left(q_{0}, \dot{q}_{0}, t_{0}\right)$ in the extended phase space (or initial data space) there is a unique solution $q(t)$ passing through $q_{0}$ with velocity $\dot{q}_{0}$ for $t=t_{0}$.

Something similar happens for regular $n$th order Lagrangians, whose Euler-Lagrange equations yield a $2 n$th order ordinary differential system.

Thus, the existence and uniqueness theorems ${ }^{8}$ play a crucial role in the definition of the phase space for local systems. Moreover, since these theorems establish a 1 to 1 correspondence between the phase space and the space of solutions to the Euler-Lagrange equations, we could readily take both spaces as equivalent objects from the mathematical point of view. ${ }^{9}$

In the nonlocal case the Euler-Lagrange equations are of functional-differential type (integrodifferential or difference-differential in the best cases). In most instances there is nothing similar to existence and uniqueness theorems, no finite set of initial data is enough to determine a solution and it is not at all clear what the initial data space is like.

Most approaches, ${ }^{4,10-12}$ to the construction of a Hamiltonian formalism for nonlocal Lagrangians have tried to change the functional part of the Euler-Lagrange equations into something more handy, by expressing it as a function of all the derivatives of any order of $q(t)$. These equations thus become local, but only in appearance. Then, either the problem of defining a finite dimensional phase space is avoided ${ }^{12}$ and the Hamiltonization procedure ends with a sort of extension of Ostrogradski transformation, ${ }^{13}$ or a finite dimensional phase space is defined for perturbative solutions, ${ }^{4,11,14}$ only.

Since the latter methods are either cumbersome and sometimes depend crucially on the form of the perturbative expansion of the Lagrangian, a few years ago we developed a new way that does not $\mathrm{mix}$ the Hamiltonization procedure with the perturbative expansions. ${ }^{15}$ Although that procedure is perfectly valid and does not differ essentially from the methods exposed in the 
present paper, its presentation there was mainly based on guess work. Moreover, this paper had strong overtones of the relativistic systems of directly interacting particles-the topic that the method had been devised for-which may make it less accessible to scholars interested in other fields in theoretical physics.

To provide a clearer idea of the above mentioned initial data problem, let us have a more detailed look at Fokker-Wheeler-Feynman electrodynamics ${ }^{1}$ for two point charges. The Lagrangian is:

$$
L=-m_{1} \sqrt{-\dot{x}_{1}^{2}}-m_{2} \sqrt{-\dot{x}_{2}^{2}}+e_{1} e_{2} \int_{\mathbf{R}} d \tau^{\prime} \delta\left(\left[x_{1}-x_{2}\left(\tau^{\prime}\right)\right]^{2}\right) \dot{x}_{1} \dot{x}_{2}\left(\tau^{\prime}\right)
$$

and the Euler-Lagrange equations are

$$
m_{a} \ddot{x}_{a}(\tau)=\frac{e_{a}}{2}\left[F_{b}\left(\tau_{b}^{\mathrm{ret}}, x_{a}\right) \dot{x}_{a}(\tau)+F_{b}\left(\tau_{b}^{\mathrm{adv}}, x_{a}\right) \dot{x}_{a}(\tau)\right]
$$

where $F_{b}$ is the electric field produced by charge $b$, and $a \neq b-1,2$.

So, to determine the acceleration of charge 1 at proper time $\tau$, we must know the state of charge 2 at $\tau_{2}^{\text {ret }}$ and $\tau_{2}^{\text {adv }}$. As $\tau$ elapses, we need data that belong more and more to the future of worldline 2 to determine the acceleration of 1 . One could think that giving $x_{2}(\tau)$ for all $\tau$ in the right hand side, Eq. ( 2) with $a=1$ becomes a second order ordinary differential equation that determines $x_{1}(\tau)$ for any given initial position $x_{1}\left(\tau_{0}\right)$ and velocity $\dot{x}_{1}\left(\tau_{0}\right)$. We must then substitute this $x_{1}(\tau)$ in Eq. (2) for $a=2$ and, most probably, the first given $x_{2}(\tau)$ will not be a solution.

To summarize a little roughly: if we are given less than the whole solution, then probably the initial data will be too little. But if the whole solution is given as initial data, it will be too much.

The above comments suggest we should change our attitude towards the Euler-Lagrange equations and consider them as constraints on the space of all the possible solutions $\mathscr{Q}^{\prime}\left(\mathbf{R}, M_{4} \times M_{4}\right)=\left\{q(\tau), q=\left(x_{1}^{\mu}, x_{2}^{\nu}\right)\right\}$, rather than as evolution equations. (The latter is only a nice particular feature of the local case that comes from the existence and uniqueness theorems for ordinary differential equations.)

The present paper is organized as follows. In Sec. II we state the variational principle for a nonlocal Lagrangian system, derive the corresponding Euler-Lagrange equations and define its phase space, $\Gamma$. In Sec. III we introduce an equivalent local first order singular Lagrangian, whose standard Hamiltonian formalism lives in a larger phase space $\Gamma^{E}$, and establish the relationship between the above Euler-Lagrange equations and the Hamilton equations plus a constraint. The very important notion of reduction of finite order ${ }^{16}$ is introduced in Sec. IV as a subcollection of the set of solutions, and the reduced phase space $\Gamma^{R}$ is defined. In Sec. V the Hamiltonian formalism is referred to a symplectic form on $\Gamma^{E}$, which is pulled back onto the reduced phase space to yield a Hamiltonian formalism there. In Sec. VI we first apply our methods to Lagrangians that are either local or can be derived from other local Lagrangians-i.e., by formally intcgrating the equations for a few degrees of freedom and find the same Hamiltonian formalism we should have obtained by the standard methods (this allows us to conclude therefore that the methods proposed here are a generalization of the standard ones). In Sec. VI C we finally present the application of our methods to systems that are truly nonlocal and admit local perturbative expansions. The latter case is very common in theoretical physics where a Lagrangian consisting of a local term plus a nonlocal one times a coupling constant is often found. Special attention is paid to some Lagrangians cited in Ref. 4 in connection with string field theory. 


\section{NONLOCAL LAGRANGIAN SYSTEMS}

A nonlocal Lagrangian is defined by a functional $L([q])$ that depends on a continuous vector function $q^{l}(\lambda), \lambda \in \mathbb{R}$ and $l=1, \ldots, N$. We shall also assume some conditions on the behavior of $q^{l}(\lambda)$ for $\lambda \rightarrow \pm \infty$.

The action principle associated to this Lagrangian reads

$$
\delta S \equiv \delta \int_{\mathbf{R}} L(t) d t=0
$$

for any $\delta q^{l}(\lambda)$ with compact support. Here $L(t)$ is a shorthand for $L\left(\left[T_{t} q^{l}\right]\right)$ where $T_{t} q^{l}(\lambda) \equiv q^{l}(t+\lambda)$.

Writing the functional derivative of $L$ as

$$
E_{l}\left(t, t^{\prime} ;[q]\right) \equiv \frac{\delta L(t)}{\delta q^{\prime}\left(t^{\prime}\right)}
$$

the action principle (3) yields the Euler-Lagrange equation

$$
\int_{\mathrm{R}} d t E_{l}\left(t, t^{\prime},[q]\right)=0
$$

Notice that the instances where $L$ is a local (standard) Lagrangian of the $n$th order are also included in the framework here developed. Indeed, we only have to take a function $L\left(q^{i}, \dot{q}^{j}, \ldots, q^{(n)}\right)$ of $N \times(n+1)$ variables and write

$$
L(t)=L\left(q^{i}(t), \dot{q}^{j}(t), \ldots, \stackrel{(n)}{q} l(t)\right)
$$

Then

$$
E_{l}\left(t, t^{\prime} ;[q]\right)=\sum_{m=0}^{n}\left(\frac{\partial L}{\partial q^{(m)}}\right)_{(t)} \delta^{(m)}\left(t-t^{\prime}\right)
$$

which, substituted into (5) yields

$$
\sum_{m=0}^{n}\left(-\frac{d}{d t}\right)^{m}\left(\frac{\partial L}{\partial q^{(m)}}\right)=0
$$

i.e., the Euler-Lagrange equations for a Lagrangian system of the $n$th order (see Ref. 13, p. 266).

Since the integrals in (3) and (5) run through the whole $\mathbb{R}$, the action $S$ will be divergent unless $L(t)$ behaves properly at $t= \pm \infty$ for all $q^{l}(t)$ considered. However, even though $S$ is divergent, the Euler-Lagrange equations (5) could be meaningful. Indeed, we only need that $E_{l}\left(t, t^{\prime} ;[q]\right)$ is summable on $t \in \mathbb{R}$, for any given $t^{\prime} \in \mathbf{R}$.

This is the case for a wide variety of nonlocal Lagrangians that have physical interest, where $E_{l}\left(t, t^{\prime}\right)$ decreases very fast as $\left|t-t^{\prime}\right|$ increases. Therefore, we shall hereafter assume that there is a rapidly increasing function $N(t)$ such that

$$
\lim _{\left|t-t^{\prime}\right| \rightarrow \infty} E_{l}\left(t, t^{\prime}\right) N\left(t-t^{\prime}\right)=0 .
$$


This is indeed the case for local Lagrangians because, as we have seen in (6), $E\left(t, t^{\prime}\right)$ is a sum of $\delta$-functions and its derivatives, or in the nonlocal examples examined in Secs. VI and VI C. We shall now denote by $\Gamma$ the collection of solutions $q^{l}(t), l=1, \ldots, N$, to the Euler-Lagrange equations (5) and call it the phase space of the nonlocal Lagrangian system.

Equations (5) are usually of functional-differential type and, generally, there is no theorem of existence and uniqueness for this kind of functional equations, therefore the phase space $\Gamma$ cannot be parametrized as easily as in local cases. Generally it is not possible to find a finite set of parameters characterizing every given solution in $\Gamma$. The latter rule obviously fails in local instances, and there is also a variety of nonlocal cases where it also does fail, as we shall see in Secs. VI and VI C.

\section{THE EQUIVALENT FIRST ORDER LAGRANGIAN}

We now consider the Lagrangian system

$$
\tilde{L}(t)=L([Q(t, \lambda)])+\int_{\mathrm{R}} d \lambda \mu_{l}(t, \lambda)\left[\dot{Q}^{l}(t, \lambda)-{Q^{\prime}}^{l}(t, \lambda)\right]
$$

where' means partial derivative with respect to $\lambda$, and $L$ is a functional on $Q^{l}(t, \lambda)$ and is considered local in the evolution parameter $t$ but nonlocal in $\lambda$.

Notice that we are somehow replacing $q^{l}(t)$ by a function of two independent variables $Q^{l}(t, \lambda)$, but we immediately add the second term on the right hand side suggesting a constraint $\partial_{t} Q-\partial_{\lambda} Q=0$ [i.e., $Q(t, \lambda)$ depends only on the variable $\left.t+\lambda\right]^{17}$

The new Lagrangian $\tilde{L}(t)$ can be viewed as first order Lagrangian for a $1+1$ dimensional field theory, $\left(Q^{l}(t, \lambda), \mu_{j}(t, \lambda)\right) ; l, j=1, \ldots, N$, that is local in the time variable, $t$, but nonlocal in the "space" variable, $\lambda$. Thus, since it is local in time, we shall apply the standard Hamiltonian formalism to $\tilde{L}$ and then translate it into our temporally nonlocal case.

To ensure the convergence of the last term on the right hand side of (8), for the admitted functions $Q^{l}(t, \lambda)$ some restrictions must be imposed on $\mu_{l}(t, \lambda)$.

First of all we shall assume that $Q^{l}(t, \lambda)$ is continuous, has continuous derivatives and does not increase faster than $N(\lambda) \cdot \lambda^{-\alpha}, \alpha>2$, as $|\lambda| \rightarrow \infty$. That is,

$$
\lim _{|\lambda| \rightarrow \infty} \frac{\left|Q^{l}(t, \lambda)\right|}{N(\lambda) \lambda^{-\alpha}}=0 ; \quad \alpha>2 .
$$

Furthermore, we shall require that $\mu_{l}(t, \lambda)$ is such that

$$
\int_{R} \mu_{l}(t, \lambda) \varphi(t, \lambda)<+\infty
$$

for any continuous function $\varphi(t, \lambda)$ fulfilling the condition (9).

The Euler-Lagrange equations for $\tilde{L}$

$$
\begin{gathered}
\dot{Q}^{\prime}(t, \lambda)-Q^{\prime l}(t, \lambda)=0, \\
\frac{\delta L(t)}{\delta Q^{l}(t, \lambda)}-\dot{\mu}_{l}(t, \lambda)+\mu_{l}^{\prime}(t, \lambda)=0,
\end{gathered}
$$

are first order partial differential equations rather than second order, because $\tilde{L}$ is singular.

The first term on the left of Eq. (12) must be understood as 


$$
\frac{\delta L(t)}{\delta Q^{l}(t, \lambda)} \equiv \frac{\delta L([Q(t, \lambda])}{\delta Q^{l}(t, \lambda)} .
$$

Moreover, since $\tilde{L}$ is singular, the Legendre transformation

$$
\begin{gathered}
P_{l}(t, \lambda)=\frac{\delta L(t)}{\delta \dot{Q}^{l}(t, \lambda)}=\mu_{l}(t, \lambda), \\
\Pi^{l}(t, \lambda)=\frac{\delta L(t)}{\delta \dot{\mu}_{l}(t, \lambda)}=0,
\end{gathered}
$$

is degenerate and the phase space of our system is not the whole space spanned by

$$
\left(Q^{l}\left(t, \lambda_{1}\right), \mu_{j}\left(t, \lambda_{2}\right), P_{i}\left(t, \lambda_{3}\right), \Pi^{k}\left(t, \lambda_{4}\right)\right)
$$

but only the submanifold defined by the primary constraints

$$
\phi_{l}(t, \lambda) \equiv P_{l}(t, \lambda)-\mu_{l}(t, \lambda) \approx 0 ; \quad \chi^{i}(t, \lambda) \equiv \Pi^{i}(t, \lambda) \approx 0 .
$$

Moreover, the Hamiltonian is

$$
H=\int_{\mathrm{R}} d \lambda P_{l}(t, \lambda) Q^{\prime \prime}(t, \lambda)-L([Q(t, \lambda)])+\int_{\mathrm{R}} d \lambda\left[a^{l}(t, \lambda) \phi_{l}(t, \lambda)+b_{l}(t, \lambda) \chi^{l}(t, \lambda)\right],
$$

where $a^{l}(t, \lambda)$ and $b_{l}(t, \lambda)$ are Lagrange multipliers.

The primary constraints (15) are second class

$$
\left\{\phi_{l}(t, \lambda), \chi^{i}\left(t, \lambda^{\prime}\right)\right\}=-\delta_{l}^{i} \delta\left(\lambda-\lambda^{\prime}\right) ;\left\{\phi_{l}(t, \lambda), \phi_{j}\left(t, \lambda^{\prime}\right)\right\}=\left\{\chi^{l}(t, \lambda), \chi^{j}\left(t, \lambda^{\prime}\right)\right\}=0 .
$$

Hence, the Dirac theory of constraints can be applied to eliminate the variables $\mu$ and $\Pi$. The elementary Dirac brackets for the remaining variables are

$$
\left\{Q^{i}(t, \lambda), P_{j}\left(t, \lambda^{\prime}\right)\right\}_{D}=\delta_{j}^{i} \delta\left(\lambda-\lambda^{\prime}\right) ;\left\{Q^{i}(t, \lambda), Q^{j}\left(t, \lambda^{\prime}\right)\right\}_{D}=\left\{P_{i}(t, \lambda), P_{j}\left(t, \lambda^{\prime}\right)\right\}_{D}=0 .
$$

The Hamiltonian is

$$
H_{D}=\int_{\mathrm{R}} d \lambda P_{l}(t, \lambda) Q^{\prime l}(t, \lambda)-L(t)
$$

and the Hamilton-Dirac equations are

$$
\begin{gathered}
\dot{Q}^{l}(t, \lambda)=\frac{\delta H_{D}}{\delta P_{l}(t, \lambda)}=Q^{\prime l}(t, \lambda), \\
\dot{P}_{l}(t, \lambda)=-\frac{\delta H_{D}}{\delta Q^{l}(t, \lambda)}=P_{l}^{\prime}(t, \lambda)+\frac{\delta L(t)}{\delta Q^{l}(t, \lambda)},
\end{gathered}
$$

Now, constraint (15) and condition (12) on $\mu_{l}(t, \lambda)$ imply that

$$
\int_{\mathrm{R}} P_{l}(t, \lambda) \cdot \varphi(t, \lambda)<+\infty
$$

for all continuous functions $\varphi(t, \lambda)$ fulfilling the condition (9). 
This condition is essential to ensure the convergence of $H_{D}$ and to derive Eq. (21) for $\dot{P}_{l}(t, \lambda)$.

The Hamilton-Dirac equations (20), (21) can be easily integrated, so obtaining

$$
\begin{gathered}
Q^{l}(t, \lambda)=Q^{l}(0, t+\lambda), \\
P_{l}(t, \lambda)=\frac{1}{2} \int d \sigma \operatorname{sign}(\sigma) \frac{\delta L(t-\sigma)}{\delta Q^{l}(t-\sigma, \lambda+\sigma)}+K_{l}(t+\lambda),
\end{gathered}
$$

where $K_{l}$ is an arbitrary function of $t+\lambda$.

This, in terms of initial data leads to the following.

Proposition 1: Given $N$-functions $\zeta^{l}(\lambda) ; l=1, \ldots, N, \lambda \in \mathbb{R}$ that are continuous with continuous derivatives and fulfill the slow growth condition (9), the functions

$$
\begin{gathered}
Q^{l}(t, \lambda)=\zeta^{l}(t+\lambda), \\
P_{l}(t, \lambda)=\frac{1}{2} \int_{\mathrm{R}} d \sigma \operatorname{sign}(\sigma) E_{l}(t-\sigma, t+\lambda,[\zeta])+K_{l}(t+\lambda),
\end{gathered}
$$

are a solution of the Hamilton-Dirac equations (20) and (21) for any arbitrary functions $K_{l}(t+\lambda)$; $l=1, \ldots, N$, and with $E_{l}\left(t, t^{\prime},[\zeta]\right)$ given by $(4)$.

The result (25), (26) follows immediately from (23) and (24), and the fact that

$$
\left.\frac{\delta L(t-\sigma)}{\delta Q(t-\sigma, \lambda+\sigma)}\right|_{Q\left(t^{\prime}, \lambda^{\prime}\right)=\zeta\left(t^{\prime}+\lambda^{\prime}\right)}=\frac{\delta L\left(\left[T_{t-\sigma} \zeta\right]\right)}{\delta \zeta(t+\lambda)}=E(t-\sigma, t+\lambda,[\zeta]) .
$$

Equation (26) can also be written as:

$$
P_{l}(t, \lambda)=\int_{\mathbf{R}} d \sigma \chi(\lambda, \sigma) E_{l}(t-\sigma, t+\lambda,[\zeta])+K_{l}(t+\lambda)-\frac{1}{2} \operatorname{sign}(\lambda) \int_{\mathbf{R}} d \sigma E_{l}(\sigma, t+\lambda,[\zeta]),
$$

with

$$
\chi(\lambda, \sigma)=\frac{1}{2}(\operatorname{sign}(\sigma)+\operatorname{sign}(\lambda))=\left\{\begin{array}{lc}
1 & \lambda>0, \sigma>0 \\
-1 & \lambda<0, \sigma<0 \\
0 & \text { otherwise }
\end{array}\right.
$$

We should now check whether the summability condition (22) is fulfilled. In Appendix A this condition is proven to hold for the first two terms on the right hand side of Eq. (28). Moreover, it is obvious that this summability condition holds for the last term, at least for $\zeta(\lambda)$ in a neighborhood of the solutions of the Euler-Lagrange equations. [Indeed, notice that this term vanishes if $Q(0, \lambda)=\zeta(\lambda)$ is a solution of Eq. (5).]

As a consequence, we arrive at the following result.

Proposition 2: For any given solution $q^{l}(t) ; l=1, \ldots, N$ of the Euler-Lagrange equations (5) there is a solution $\left(Q^{l}(t, \lambda), P_{l}(t, \lambda)\right) ; l=1, \ldots, N$ of the Hamilton equations (20), (21) fulfilling the convergence conditions (22).

Proof: The functions $Q^{l}$ and $P_{l}$ are obtained from (25) and (28), writing $q^{l}$ instead of $\zeta^{l}$, taking $K_{l}=0$ and using the fact that $q^{l}$ is a solution of (5). [Notice that the canonical transformation with generating function $F(\tilde{Q}, P, t) \equiv \int d \lambda \tilde{Q}^{l}(t, \lambda)\left[-P_{l}(t, \lambda)+K_{l}(t+\lambda)\right]$ transforms $\tilde{Q}^{l}(t, \lambda)$ into $Q^{l}(t, \lambda)$ and $\tilde{P}_{l}(t, \lambda)$ into $P_{l}(t, \lambda)+K_{l}(t+\lambda)$.] We can therefore write: 


$$
\begin{gathered}
Q^{l}(t, \lambda)=q^{l}(t+\lambda), \\
P_{l}(t, \lambda)=\int d \sigma \chi(\lambda, \sigma) E_{l}(t-\sigma, t+\lambda,[q]) .
\end{gathered}
$$

This enables us to establish a 1 to 1 correspondence between the phase space $\Gamma$ (with infinitely many dimensions) of the Euler-Lagrange equations (5) and a submanifold of the phase space $\Gamma^{E}$ of the Hamilton equations (20) and (21):

$$
j_{E}: \Gamma \rightarrow \Gamma^{E}, \quad q^{l}(\lambda) \rightarrow\left(Q^{l}(0, \lambda), P_{j}(0, \lambda)\right)
$$

with

$$
Q^{l}(0, \lambda)=q^{l}(\lambda), \quad P_{j}(0, \lambda)=\int d \sigma \chi(\lambda, \sigma) E_{j}(-\sigma, \lambda ;[q])
$$

It can be easily proven that the map $j_{E}$ commutes with time evolution.

Indeed, time evolution on $\Gamma^{E}$ is defined by the Hamiltonian flow of (20), (21)

$$
T_{t}^{E}\left(Q^{l}(0, \lambda), P_{j}(0, \lambda)\right)=\left(Q^{l}(t, \lambda), P_{j}(t, \lambda)\right),
$$

where, according to (25) and (28)

$$
\begin{gathered}
Q^{l}(t, \lambda)=Q^{l}(0, t+\lambda) \equiv q^{l}(t+\lambda), \\
P_{j}(t, \lambda)=\int d \sigma \chi(\lambda, \sigma) E_{j}(t-\sigma, t+\lambda ;[q])+K_{j}(t+\lambda) \\
+\frac{1}{2} \operatorname{sign}(\lambda) \int d \sigma E_{j}(\sigma, t+\lambda ;[q]) .
\end{gathered}
$$

Choosing the initial data (31), taking into account that $q^{l} \in \Gamma$ and writing $q^{\prime \prime}$ for $T_{t} q^{l}$, we obtain $K_{l}=0$ and arrive at

$$
\begin{aligned}
Q^{l}(t, \lambda)=q^{l}(t+\lambda)=T_{t} q^{l}(\lambda)=q^{\prime l}(\lambda), \\
P_{j}(t, \lambda)=\int d \sigma \chi(\lambda, \sigma) E_{j}(t-\sigma, t+\lambda ;[q]) \\
=\int d \sigma \chi(\lambda, \sigma) \frac{\delta L\left(\left[T_{t-\sigma} q\right]\right)}{\delta q^{j}(t+\lambda)} \\
=\int d \sigma \chi(\lambda, \sigma) \frac{\delta L\left(\left[T_{-\sigma} q^{\prime}\right]\right)}{\delta q^{\prime j}(\lambda)} \\
=\int d \sigma \chi(\lambda, \sigma) E_{j}\left(-\sigma, \lambda ;\left[q^{\prime}\right]\right),
\end{aligned}
$$

which, again using (31), leads to

$$
\forall q^{l} \in \Gamma ; \quad T_{t}^{E} \circ j_{E}\left(q^{l}\right)=j_{E^{\circ}} T_{t}\left(q^{l}\right)
$$




\section{REDUCTIONS OF FINITE ORDER}

For certain Lagrangians, by applying a variety of procedures (see Sec. VI C below), it is possible to find an ordinary differential system of finite order:

$$
\stackrel{(r)}{q}^{k}=\xi^{k}\left(q^{i}, \dot{q}^{j}, \ldots, \stackrel{(r-1)}{q} l\right), i, j, k, l, \ldots=1, \ldots, N
$$

such that every solution of (35) is a solution the Euler-Lagrange equations (5). [The latter does not mean the converse, namely, there are many solutions of (5) that do not obey (35).] In such a case we say that (35) is an r-order reduction of the functional differential equations (5).

Let $\varphi^{l}(t, z)$ be a solution of (35) for some given initial data $z=\left(q_{0}^{i}, \dot{q}_{0}^{j}, \ldots, r^{-1} l_{0}\right)$ and let $\Gamma^{R}$ denote the space of initial data (phase space) of the r-order reduction (35). We can then establish the 1 to 1 map

$$
\begin{gathered}
j_{R}: \Gamma^{R} \rightarrow \Gamma, \\
z \rightarrow q^{l}(\lambda)=\varphi^{l}(\lambda, z),
\end{gathered}
$$

because, Eqs. (35) being reduction of (5), every solution the former is also a solution of the latter and hence $\varphi^{l}(\lambda, z) \in \Gamma$.

Proposition 3: The map $j_{R}$ commutes with time evolution.

Proof: We have a time evolution transformation $T_{t}^{R}: \Gamma^{R} \rightarrow \Gamma^{R}$ defined by

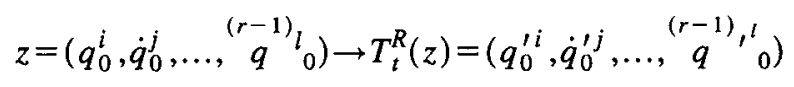

with

$$
\stackrel{(s)}{q},{ }_{0}=\left[\frac{\partial^{s} \varphi^{l}(t, z)}{\partial t^{s}}\right]_{t=t_{0}},
$$

where $\varphi^{l}(t, z)$ is the solution of (35) determined by the initial data $z \in \Gamma^{R}$.

Furthermore, we have also a time evolution acting on $\Gamma$ :

$$
\begin{gathered}
T_{t}: \Gamma \rightarrow \Gamma, \\
q^{l}(\lambda) \rightarrow\left(T_{t} q^{l}\right)(\lambda)=q^{l}(t+\lambda) .
\end{gathered}
$$

By the theorem of existence and uniqueness of solutions, ${ }^{8}$ we have for the ordinary differential system (35) that

$$
\forall z \in \Gamma^{R}, t, \lambda \in \mathbb{R}, \quad \varphi^{l}(t+\lambda, z)=\varphi^{l}\left(\lambda, T_{t}^{R} z\right)
$$

which, using (36) and (39), can be written as

$$
\forall z \in \Gamma^{R} ; \quad T_{t}^{\circ} j_{R}(z)=j_{R}^{\circ} T_{t}^{R}(z),
$$

which proves the proposition.

Now recalling the 1-to-1 map $j_{E}: \Gamma \rightarrow \Gamma^{E}$ introduced at the end of Sec. III, we find that the product

$$
j=j_{E^{\circ}} j_{R}
$$

is a 1-to-1 map from $\Gamma^{R}$ into $\Gamma^{E}$, and from (41) and (34) we also have 


$$
\forall z \in \Gamma^{R} ; \quad j \circ T_{t}^{R}(z)=T_{t}^{E} \circ j(z)
$$

Moreover, the following diagram is commutative

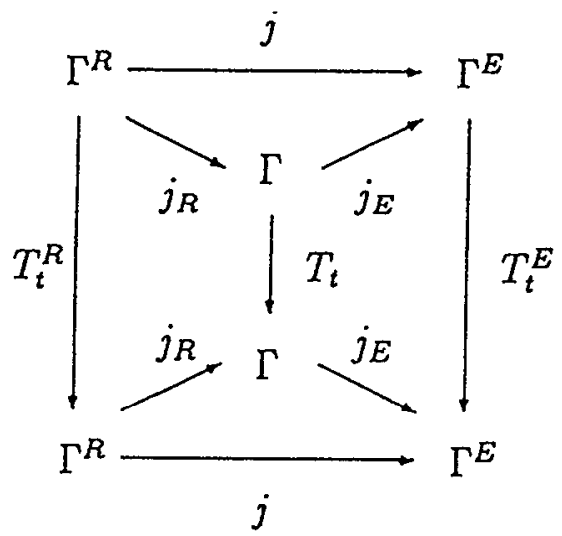

As a consequence of this commutativity, the infinitesimal generators of the various time evolutions, $T^{R}, T$, and $T^{E}$, are related by the respective Jacobian mappings.

At every given $\left.z=\left(q^{i}, \dot{q}^{j}, \ldots, \stackrel{(r}{q}^{l}{ }^{l}\right)\right) \in \Gamma^{R}$ the infinitesimal generator ${ }^{18}$ of $T^{R}$ is the vector $\mathbf{X}_{z}^{R}$ tangent to the orbit $\left\{T_{t}^{R}(Z) ; t \in \mathbb{R}\right\}$ at $t=0$. According to (38) and (35), we have that

$$
\mathbf{X}_{z}^{R}=\dot{q}^{l} \frac{\partial}{\partial q^{l}}+\ddot{q}^{l} \frac{\partial}{\partial \dot{q}^{l}}+\ldots+\stackrel{(r-1)}{q} \frac{\partial}{\underset{(r-2)}{l}+\xi^{l}}+\xi^{l}(q, \ldots, \stackrel{(r-1)}{q}) \frac{\partial}{(r-1)},
$$

which acts on functions defined on $\Gamma^{R}$.

Similarly, the infinitesimal generator of $T$ at $q^{l} \in \Gamma$ is

$$
\mathbf{X}_{q}=\int_{\mathbf{R}} d \lambda q^{\prime}{ }_{l}(\lambda) \frac{\delta}{\delta q^{l}(\lambda)}
$$

which acts on functional defined on $\Gamma$.

Finally the generator of $T^{E}$ at $(Q, P) \in \Gamma^{E}$ is

$$
\mathbf{X}_{(Q, P)}^{E}=\int_{\mathrm{R}} d \lambda\left\{\dot{Q}^{l}(t, \lambda) \frac{\delta}{\delta Q^{l}(t, \lambda)}+\dot{P}_{j}(t, \lambda) \frac{\delta}{\delta P_{j}(t, \lambda)}\right\},
$$

with $\dot{Q}^{l}$ and $\dot{P}_{j}$ given by the Hamilton Eqs. (20) and (21).

As a consequence of (41), (34), and (42) we have respectively that

$$
\begin{aligned}
& \mathbf{X}_{j_{R}}=d j_{R}\left(\mathbf{X}_{z}^{R}\right) ; \forall z \in \Gamma^{R}, \\
& \mathbf{X}_{{ }_{j_{E}}}^{E}=d j_{E}\left(\mathbf{X}_{q}\right) ; \forall q \in \Gamma, \\
& \mathbf{X}_{j z}^{E}=d j\left(\mathbf{X}_{z}^{R}\right) ; \forall z \in \Gamma^{R} .
\end{aligned}
$$




\section{HAMILTONIAN FORMALISM}

The generator of time evolution, $\mathbf{X}^{E}$, on $\Gamma^{E}$ is defined by the Hamilton Eqs. (20), (21). We can write the latter in more compact a fashion as the Hamiltonian vector field associated to the Hamilton function $H_{D}$ by a symplectic form. ${ }^{19}$

The latter is the nonsingular, closed differential two-form on $\Gamma^{E}$

$$
\Omega^{E}=\int_{\mathbf{R}} d \lambda \delta P_{l}(0, \lambda) \wedge \delta Q^{l}(0, \lambda) \in \Lambda^{2}\left(\Gamma^{E}\right)
$$

Notice that two distinct symbols for differential occur in (48), in order to distinguish $d \lambda$ from $\delta Q$ and $\delta P$, the differential of the integration parameter $\lambda$ and the functional differentials of dynamical variables, respectively.

The Hamilton Eqs. (20), (21) can then be expressed as

$$
i\left(\mathbf{X}^{E}\right) \Omega^{E}=-\delta H_{D}
$$

The pull back mapping ${ }^{20}$ corresponding to $j: \Gamma^{R} \rightarrow \Gamma^{E}$ is

$$
d j: \Lambda^{2}\left(\Gamma^{E}\right) \rightarrow \Lambda^{2}\left(\Gamma^{R}\right)
$$

which enables us to define a differential 2-form $\forall z \in \Gamma^{R}$

$$
\Omega_{z}^{R} \equiv \operatorname{dj}\left(\Omega_{j z}^{E}\right)
$$

Now, since $\Omega^{E}$ is closed and the pull back commutes with the exterior derivative, ${ }^{20} \Omega^{R}$ is also closed. However, $\Omega^{R}$ may be singular and hence it need not be symplectic. It is then said to be presymplectic.

Due to the properties of the pull back mapping $d j,{ }^{20}$ we have that $\forall z \in \Gamma^{R}$

$$
\begin{aligned}
& i\left(\mathbf{X}_{z}^{R}\right) \Omega_{z}^{R}=i\left(\mathbf{X}_{z}^{R}\right)\left(d j \Omega_{j z}^{E}\right)=d j\left[i\left(d j \mathbf{X}_{z}^{R}\right)\left(\Omega_{j z}^{E}\right)\right]= \\
& d j\left[i\left(\mathbf{X}_{j z}^{E}\right) \Omega_{j z}^{E}\right]=d j\left(-\delta H_{D}\right)_{j z}=-\delta\left(j^{*} H_{D}\right)_{z},
\end{aligned}
$$

where (47) and (49) have been used.

Then, writing

$$
H^{R} \equiv j^{*} H_{D}=H_{D^{\circ}} j
$$

we arrive at

$$
i\left(\mathbf{X}^{R}\right) \Omega^{R}=-\delta H^{R}
$$

that is, $\mathrm{X}^{R}$ is a Hamiltonian vector field on $\Gamma^{R}$ associated to the Hamiltonian function $H^{R}$ by the presymplectic form $\Omega^{R}$.

If $\Omega^{R}$ is nonsingular, it enables us to set up a Poisson bracket formalism for the generator $\mathrm{X}^{R}$, i.e., the reduced system ( 35 ). This we shall see in the next section.

An explicit expression for $\Omega^{R}$ can be obtained by substituting $q^{l}(\lambda)$ for $\varphi^{l}(\lambda, z)$ in (31)

$$
P_{l}(0, \lambda ; z)=\int d \sigma \chi(\lambda, \sigma) E_{j}(-\sigma, \lambda ;[\varphi])
$$

and in (48) 


$$
\Omega^{R}=\int d \lambda \int d \sigma \int d \rho \chi(\lambda, \sigma) \frac{\delta E_{j}(-\sigma, \lambda ;[\varphi])}{\delta q^{k}(\rho)} \delta \varphi^{k}(\rho, z) \wedge \delta \varphi^{j}(\lambda, z)
$$

with

$$
\chi(\lambda, \sigma)=\frac{1}{2}(\operatorname{sign}(\sigma)+\operatorname{sign}(\lambda))
$$

and

$$
z \equiv\left(q^{i}, \dot{q}^{j}, \ldots,{ }^{(r-1)}{ }^{l}\right) .
$$

Moreover, replacing (31) in (19), we obtain an explicit expression for the Hamiltonian

$$
H^{R}=\int d \lambda \int d \sigma \chi(\lambda, \sigma) E_{j}(-\sigma, \lambda ;[\varphi]) \dot{\varphi}^{j}(\lambda, z)-L([\varphi(\lambda, z)])
$$

\section{APPLICATIONS}

In the first and second cases here considered, the phase space $\Gamma$ has finitely many dimensions and $\Gamma^{R}=\Gamma$ can be taken.

\section{A. Local Lagrangians}

Let us consider a local Lagrangian, depending on the coordinates $q^{\alpha}$ and their derivatives up to the $n$th order, all the same instant $t$ :

$$
L(t)=L\left(q^{\alpha}(t), \dot{q}^{\beta}(t), \ldots,{ }_{q}^{(n)}{ }^{\nu}(t)\right), \quad \alpha, \beta, \ldots, \nu=1, \ldots, m
$$

In this case

$$
E_{\alpha}\left(t^{\prime}, t ;[q]\right)=\sum_{r=0}^{n}\left(\frac{\partial L}{\underset{(r)}{\partial q^{\alpha}}}\right)_{(t)} \delta^{(r)}\left(t^{\prime}-t\right)
$$

and the Euler-Lagrange Eqs. (5) read

$$
\mathscr{E}_{\alpha}(t ;[q])=\sum_{r=0}^{n}(-1)^{r} \frac{d^{r}}{d t^{r}}\left(\begin{array}{c}
\frac{\partial L}{(r)} \\
\partial q^{\alpha}
\end{array}\right)_{(t)}=0 .
$$

If $L$ is nonsingular, that is,

$$
\operatorname{det}\left(\frac{\partial^{2} L}{\partial(n) q^{\alpha} \partial_{q}^{\beta} \beta}\right)_{\alpha, \beta=1, \ldots, m} \neq 0
$$

then Eqs. (58) can be solved for the derivatives $\stackrel{(2 n)}{q} \alpha$ and for every set of initial data $\left(t_{0}, q^{\alpha}, \ldots, q^{(2 n-1)} \nu\right)$, there is one solution $\zeta^{\alpha}(t)$ such that 


$$
\mathscr{E}_{\alpha}(t ;[\zeta])=0
$$

and

$$
\frac{d^{r} \zeta^{\alpha}\left(t_{0}\right)}{d t^{r}}=\stackrel{(r)}{q}^{\alpha} ; r=0,1, \ldots, 2 n-1, \alpha=1, \ldots, m .
$$

Therefore the phase space, $\Gamma$, of all solutions of (58) can be coordinated by $\left(q^{\alpha}, \dot{q}^{\beta}, \ldots, \quad(2 n-1), q^{\nu}\right)$, and $\operatorname{dim} \Gamma=2 n \times m$.

The momenta and the presymplectic form $\Omega^{R}$ can be thus calculated substituting (57) into (53) so obtaining

$$
P_{\alpha}(0, \lambda)=\sum_{1 \leqslant l \leqslant r \leqslant n}(-1)^{r+1}\left(\begin{array}{l}
r \\
l
\end{array}\right) \delta^{(l-1)}(\lambda) \frac{d^{r-l}}{d \lambda^{r-l}}\left(\frac{\partial L}{\partial q^{\alpha}}\right)_{(\lambda)},
$$

which, using well known properties of the $\delta$-function can be also written as

$$
P_{\alpha}(0, \lambda)=\sum_{k=0}^{n-1} p_{\alpha k} \delta^{(k)}(-\lambda)
$$

with

$$
p_{\alpha k} \equiv \sum_{l=0}^{n-k-1}(-1)^{l} \frac{d^{l}}{d t^{l}}\left(\frac{\partial L}{\partial \begin{array}{c}
(l+k+1) \\
q^{\alpha}
\end{array}}\right)
$$

namely, the Ostrogradski ${ }^{13}$ transformation which, by (59), is nondegenerate and hence a true transformation of coordinates.

Furthermore, using (60) in the Hamiltonian (51) we obtain

$$
H^{R}=\sum_{\alpha} \sum_{k=0}^{n-1} p_{\alpha k}{ }^{(k+1)} \alpha-L(q, \dot{q}, \ldots, \stackrel{(n)}{q})
$$

and the presymplectic form

$$
\Omega^{R}=\sum_{\alpha} \sum_{k=0}^{n-1} \delta p_{\alpha k} \wedge \delta q^{(k)}
$$

which is obviously nonsingular. These are respectively the same Hamiltonian function and symplectic form we should have obtained by applying the Ostrogradski transformation to the $n$th order Lagrangian system (56).

\section{B. Nonlocal (derived) Lagrangians}

As an illustration of a nonlocal Lagrangian whose phase space $\Gamma$ has finitely many dimensions we shall consider

$$
L(t)=\frac{1}{2} \dot{q}^{2}(t)-\frac{1}{2} q^{2}(t)+\frac{g}{4} q(t) \int_{\mathbf{R}} d t^{\prime} q\left(t^{\prime}\right) e^{-\left|t-t^{\prime}\right|}
$$


which, according to the classification in Ref. 4 belongs to the class of derived Lagrangians. Indeed,

$$
G\left(t, t^{\prime}\right)=\frac{1}{2} e^{-\left|t-t^{\prime}\right|}
$$

is the Green's function for the singular boundary value problem $\ddot{y}-y=0$, with $y(t)$ bounded for $|t| \rightarrow \infty$, and in the notation of Ref. 4 we should write

$$
\frac{g}{4} q(t) \int_{\mathrm{R}} d t^{\prime} q\left(t^{\prime}\right) e^{-\left|t-t^{\prime}\right|}=\frac{g}{2} q(t)\left(D^{2}-1\right)^{-1} q
$$

The functional derivative (4) yields

$$
E\left(t, t_{1} ;[q]\right)=\dot{q}(t) \dot{\delta}\left(t-t_{1}\right)-q(t) \delta\left(t-t_{1}\right)+\frac{g}{4}\left[\delta\left(t-t_{1}\right) \int_{\mathrm{R}} d t^{\prime} q\left(t^{\prime}\right) e^{-\left|t-t^{\prime}\right|}+q(t) e^{-\left|t-t_{1}\right|}\right]
$$

and the Euler-Lagrange Eqs. (5) are

$$
\mathscr{E}(t ;[q])=-\ddot{q}(t)-q(t)+\frac{g}{2} \int_{\mathbf{K}} d t_{1} q\left(t_{1}\right) e^{-\left|t-t_{1}\right|}=0 .
$$

To find the general solution we use that

$$
\partial_{t}^{2} G\left(t, t_{1}\right)-G\left(t, t_{1}\right)=-\delta\left(t-t_{1}\right)
$$

and substitute it into Eq. (66) to obtain

$$
q^{(i v)}-(1-g) q=0
$$

whose general solution is

$$
q(t)=\sum_{\alpha=1}^{4} A_{\alpha} e^{\omega_{\alpha} t}
$$

where $\omega_{\alpha}^{4}=1-g$, if $g \neq 1$, or

$$
q(t)=\sum_{\alpha=0}^{3} A_{\alpha} t^{\alpha}
$$

if $g=1$.

Now, since the integral in the Euler-Lagrange Eqs. (66) must be convergent, we can only consider the case $\left|\operatorname{Re}\left(\omega_{\alpha}\right)\right|<1$. For a wide range of values of $g$, the phase space $\Gamma$ can be coordinated with $(q, \dot{q}, \ddot{q}, \stackrel{(3)}{q})$ and $\operatorname{dim} \Gamma=4$

Then, using Eqs. (29), (48), and (65), we arrive at the presymplectic form

$$
\Omega^{R}=\delta \dot{q} \wedge \delta q+g \delta M \wedge \delta N .
$$

Moreover, from (51) and (29), the Hamiltonian is 


$$
H^{R}=\frac{\dot{q}^{2}}{2}+\frac{g}{2}(M \dot{N}-N \dot{M})+\frac{q^{2}}{2}-\frac{g}{4}(M+N) q
$$

with

$$
M(t)=\int_{0}^{\infty} d \lambda e^{-\lambda} q(t-\lambda) \quad \text { and } \quad N(t)=\int_{0}^{\infty} d \lambda e^{-\lambda} q(t+\lambda)
$$

Now, using (68) into these integrals, we obtain

$$
M=\frac{1}{g}(q-\dot{q}+\ddot{q}-\stackrel{(3)}{q}) \text { and } N=\frac{1}{g}(q+\dot{q}+\ddot{q}+\stackrel{(3)}{q}) .
$$

That substituted in (70) and (71) leads to

$$
\begin{gathered}
\Omega^{R}=\delta p \wedge \delta q+\delta \pi \wedge \delta x, \\
H^{R}=\frac{1}{2}\left(p^{2}+q^{2}+\pi^{2}-x^{2}-2 \sqrt{g} q \pi\right),
\end{gathered}
$$

where

$$
p=\dot{q}, \quad \pi=g^{-1 / 2}(\ddot{q}+q), \quad \text { and } \quad x=g^{-1 / 2}(\dot{q}+\stackrel{(3)}{q}) .
$$

We could have arrived at the same result by recognizing that the nonlocal Lagrangian (64) might be derived ${ }^{4}$ from the local first-order Lagrangian:

$$
L_{0}=\frac{1}{2}\left(\dot{q}^{2}+\dot{x}^{2}-q^{2}+x^{2}+2 \sqrt{g} q x\right)
$$

on replacement of $x(t)$ and $\dot{x}(t)$ by

$$
x(t)=\sqrt{g} \int_{\mathrm{R}} d t_{1} G\left(t, t_{1}\right) q\left(t_{1}\right)
$$

obtained by formally solving for $x$ the Euler-Lagrange equations derived from $L_{0}$ with the boundary conditions:

$$
x(t) \text { bounded for }|t| \rightarrow \infty
$$

\section{Perturbative expansions}

So far we have considered Lagrangians whose phase space $\Gamma$ has finitely many dimensions. Hence, an ordinary differential system that is equivalent to the Euler equations can be easily found and it itself acts as the reduction of finite order used in the methods developed in Secs. IV and V.

It often happens that the Lagrangian can be written as a sum

$$
L=L_{0}+g \cdot L_{1} \text {, }
$$

where the first term is local and the second one is nonlocal and comes multiplied by a "coupling constant", $g$.

Then, although the solutions of the Euler-Lagrange Eqs. (5) cannot usually be parametrized by a finite set of initial data, we can always find a reduction of finite order for them, which corresponds to the pertubative solutions (namely, those that can be written as a series of powers of $g$ ). ${ }^{21}$ The reduced phase space $\Gamma^{R}$ then has as many dimensions as the phase space for $L_{0}$. 
The situation is pretty similar to what happens in singular perturbation problems, ${ }^{22}$ where taking some parameter equal to zero dramatically changes the very nature of the problem. Here, taking $g=0$ converts the nonlocal Lagrangian into a local one, and the Euler-Lagrange equations become an ordinary differential system. Nevertheless, in the limit $g \rightarrow 0$, a solution of the latter equations for $L$ does not generally converge to a solution of those equations for $L_{0}$.

Let us thus consider, for instance, the Lagrangian

$$
L(t)=L_{0}(q(t), \dot{q}(t))+g L_{1}(t ;[q]),
$$

where

$$
L_{0}=\sum_{i=1}^{n} \frac{1}{2}\left(\dot{q}^{i 2}-\omega_{i}^{2} q^{i 2}\right)
$$

is a local, nonsingular, first order Lagrangian, and

$$
L_{1}=\frac{1}{3 !} \int d \lambda_{1} d \lambda_{2} d \lambda_{3} K_{i j k}\left(t-\lambda_{1}, t-\lambda_{2}, t-\lambda_{3}\right) q^{i}\left(\lambda_{1}\right) q^{j}\left(\lambda_{2}\right) q^{k}\left(\lambda_{3}\right)
$$

and $g$ is a coupling constant. We shall also assume that the kernel in (77) is symmetric, that is, for all permutations $\sigma \in S_{3}$

$$
K_{i j k}\left(\lambda_{1}, \lambda_{2}, \lambda_{3}\right)=K_{\sigma_{i} \sigma_{j} \sigma_{k}}\left(\lambda_{\sigma_{1}}, \lambda_{\sigma_{2}}, \lambda_{\sigma_{3}}\right)
$$

For further calculations it will be useful to have

$$
\begin{aligned}
E_{l}\left(t, t^{\prime} ;[q]\right)= & \frac{\delta L(t)}{\delta q^{l}\left(t^{\prime}\right)}=\dot{q}^{l} \dot{\delta}\left(t-t^{\prime}\right)-\omega_{(l)}^{2} q^{l} \delta\left(t-t^{\prime}\right) \\
& +\frac{g}{2} \int d \lambda_{1} d \lambda_{2} K_{i j l}\left(t-\lambda_{1}, t-\lambda_{2}, t-t^{\prime}\right) q^{i}\left(\lambda_{1}\right) q^{j}\left(\lambda_{2}\right)
\end{aligned}
$$

The Euler-Lagrange Eqs. (5) are thus

$$
\ddot{q}^{l}(t)+\omega_{(l)}^{2} q^{l}(t)-\frac{g}{2} \int d \lambda_{1} d \lambda_{2} d \lambda_{3} K_{i j l}\left(\lambda_{3}-\lambda_{1}, \lambda_{3}-\lambda_{2}, \lambda_{3}-t\right) q^{i}\left(\lambda_{1}\right) q^{j}\left(\lambda_{2}\right)=0,
$$

which are of integral-differential type and the problem of initial data is usually not well defined (i.e., there is no theorem of existence and uniqueness of solutions). series

For a given set of initial data $\left(q_{0}^{i}, \dot{q}_{0}^{j}\right)$, a pertubative solution of $(80)$ for these data is a power

$$
\Phi^{l}(t, z ; g)=\sum_{n=0}^{\infty} g^{n} \Phi_{(n)}^{l}(t, z)
$$

such that:

(i)

$$
\Phi_{(n)}^{l}(0, z)=q_{0}^{l} \delta_{0 n} \quad \text { and } \quad \partial_{t} \Phi_{(n)}^{l}(0, z)=\dot{q}_{0}^{l} \delta_{0 n} .
$$

(ii) The coefficients of the power series resulting from the replacement of $q(t)$ by $\Phi^{l}(t, z ; g)$ in (80) all vanish. That is 


$$
\begin{gathered}
\text { n=0 } \ddot{\Phi}_{(0)}^{l}+\omega_{l}^{2} \Phi_{(0)}^{l}(t)=0, \\
\underline{n \geqslant 1} \ddot{\Phi}_{(n)}^{l}(t, z)+\omega_{l}^{2} \Phi_{(n)}^{l}(t, z)-\frac{1}{2} \sum_{m+r=n-1} \int d \lambda_{1} d \lambda_{2} d \lambda_{3}, \\
K_{i j l}\left(\lambda_{3}-\lambda_{1}, \lambda_{3}-\lambda_{2}, \lambda_{3}-t\right) \Phi_{(m)}^{i}\left(\lambda_{1}, z\right) \Phi_{(r)}^{j}\left(\lambda_{2}, z\right)=0 .
\end{gathered}
$$

Now, (83) is a second order differential system on $\Phi_{(0)}^{l}$, and the initial data (82), determine a unique solution

$$
\Phi_{(0)}^{l}(t, z)=a_{+}^{l} e^{i \omega^{t}}+a_{-}^{l} e^{-i \omega_{l} t}
$$

with

$$
a_{ \pm}^{l}=\frac{1}{2}\left(q_{0}^{l} \mp \frac{i}{\omega_{l}} \dot{q}_{0}^{l}\right)
$$

Furthermore, every equation in the hierarchy (85), $n \geqslant 1$, can be solved once the solutions $\Phi_{(m)}^{l}(t, z)$ are known for all $m<n$. Indeed, for $n=1$, the Eq. (85) plus the initial data (82) yields, after a short manipulation,

$$
\Phi_{(1)}^{l}(t, z)=\sum_{A, B=+,-} M_{i j l}^{A B} a_{A}^{i} a_{B}^{j}
$$

with

$$
M_{i j l}^{A B}(t)=\frac{1}{2 \omega_{l}} \int_{0}^{t} d t^{\prime} \sin \omega_{l}\left(t-t^{\prime}\right) \int d \lambda_{1} d \lambda_{2} d \lambda_{3} K_{i j l}\left(\lambda_{3}-\lambda_{1}, \lambda_{3}-\lambda_{2}, \lambda_{3}-t\right) e^{i\left(A \omega_{i} \lambda_{1}+B \omega_{j} \lambda_{2}\right)}
$$

and so on for higher order coefficients $\Phi_{(n)}^{l}(t, z)$.

So far we have proven that the initial data $z=\left(q_{0}^{i}, \dot{q}_{0}^{j}\right)$ determine a unique perturbative solution of (79). Hence, we can consider the reduced phase space $\Gamma^{R}=\left\{z=\left(q_{0}^{i}, \dot{q}_{0}^{j}\right)\right\}$ and the 1 to 1 map

$$
\begin{gathered}
j_{R}: \Gamma^{R} \rightarrow \Gamma, \\
z \rightarrow \Phi^{l}(t, z ; g) .
\end{gathered}
$$

Applying the methods developed in Secs. IV and V, i.e., Eqs. (54) and (55), we then arrive at the presymplectic form

$$
\Omega^{R}(z)=\int_{\mathrm{n}^{3}} d t_{1} d t_{2} d t_{3} \chi\left(t_{1}, t_{2}\right) \frac{\delta E_{i}\left(-t_{1}, t_{2} ;\left[\Phi^{l}\right]\right)}{\delta q^{k}\left(t_{3}\right)} \delta \Phi^{k}\left(t_{3}, z ; g\right) \wedge \delta \Phi^{i}\left(t_{2}, z ; g\right)
$$

and the Hamiltonian

$$
H^{R}=\int_{\mathbf{R}^{2}} d \lambda d \sigma \chi(\lambda, \sigma) E_{j}(-\sigma, \lambda ;[\Phi]) \dot{\Phi}^{j}(\lambda, z ; g)-L([\Phi])
$$

where the kernel in (90) is obtained as the functional derivative of (80) 


$$
\begin{aligned}
\frac{\delta E_{i}\left(t_{1}, t_{2} ;[\Phi]\right)}{\delta q^{k}\left(t_{3}\right)}= & \delta_{k i}\left\{\dot{\delta}\left(t_{1}-t_{3}\right) \dot{\delta}\left(t_{1}-t_{2}\right)-\omega_{(i)}^{2} \delta\left(t_{1}-t_{2}\right) \delta\left(t_{1}-t_{3}\right)\right\} \\
& +g \int d \lambda K_{j k i}\left(t_{1}-\lambda, t_{1}-t_{3}, t_{1}-t_{2}\right) \Phi^{j}(\lambda, z ; g)
\end{aligned}
$$

Now, taking the perturbative character of $\Phi^{i}$ into account, $\Omega^{R}$ and $H^{R}$ also admit perturbative expansions

$$
\Omega^{R}=\sum_{n=0}^{\infty} g^{n} \Omega_{(n)}^{R} \quad \text { and } H^{R}=\sum_{n=0}^{\infty} g^{n} H_{(n)}^{R}
$$

The first and second terms in these expansions can be readily calculated substituting (86) into (91)

$$
\begin{gathered}
\Omega_{(0)}^{R}=\sum_{l=1}^{n} \delta \dot{q}_{0}^{l} \wedge \delta q_{0}^{l}, \\
\Omega_{(1)}^{R}=\sum_{A B C= \pm} S_{i j k}^{A B C} a_{A}^{i} \delta a_{B}^{j} \wedge \delta a_{C}^{k}, \\
H_{(0)}^{R}=\sum_{l=1}^{n} \frac{1}{2}\left(\dot{q}_{0}^{l 2}+\omega_{i}^{2} q_{0}^{l 2}\right), \\
H_{(1)}^{R}=\sum_{A B C= \pm} \frac{i C \omega_{k}}{2} S_{i j k}^{A B C} a_{A}^{i} a_{B}^{j} a_{C}^{k},
\end{gathered}
$$

with

$$
S_{i j k}^{A B C}=\frac{1}{A \omega_{i}+B \omega_{j}+C \omega_{k}} \tilde{K}_{i j k}\left(-A \omega_{i},-B \omega_{j}, A \omega_{i}+B \omega_{j}\right)
$$

and

$$
\tilde{K}_{i j k}\left(\omega_{1}, \omega_{2}, \omega_{3}\right)=\int d \lambda_{1} d \lambda_{2} d \lambda_{3} K_{i j k}\left(\lambda_{1}, \lambda_{2}, \lambda_{3}\right) e^{i\left(\omega_{1} \lambda_{1}+\omega_{2} \lambda_{2}+\omega_{3} \lambda_{3}\right)}
$$

Furthermore, the change of variables (86) enables us to rewrite $\Omega^{R}$ in terms of the variables $q^{i}, \dot{q}^{j}$.

Now, the matrix of $\Omega^{R}$ is the matrix of the elementary Lagrange brackets, whose inverse is the matrix of the elementary Poisson brackets. Hence, after a little algebra we arrive at 


$$
\begin{gathered}
\left\{q^{j}, q^{k}\right\}=\frac{-g}{4} \sum_{A B C} \frac{B C}{\omega_{j} \omega_{k}}\left(q^{l}-\frac{i A}{\omega_{(l)}} \dot{q}^{l}\right) \frac{1}{2}\left(S_{l j k}^{A B C}-S_{l k j}^{A C B}\right)+O\left(g^{2}\right), \\
\left\{q^{j}, \dot{q}^{k}\right\}=\delta_{j k}-\frac{i g}{4} \sum_{A B C} \frac{B}{\omega_{j}}\left(q^{l}-\frac{i A}{\omega_{(l)}} \dot{q}^{l}\right) \frac{1}{2}\left(S_{l j k}^{A B C}-S_{l k j}^{A C B}\right)+O\left(g^{2}\right), \\
\left\{\dot{q}^{j}, \dot{q}^{k}\right\}=\frac{g}{4} \sum_{A B C}\left(q^{l}-\frac{i A}{\omega_{(l)}} \dot{q}^{l}\right) \frac{1}{2}\left(S_{l j k}^{A B C}-S_{l k j}^{A C B}\right)+O\left(g^{2}\right),
\end{gathered}
$$

which, with a little more calculation can be seen to coincide with Eq. (3.83b) in Ref, 4.

Although it might seem at first that our methods require the previous knowledge of the solution $\Phi^{l}$ to set up the Hamiltonian formalism, this is not the case. Notice that to determine $\Omega_{(n)}^{R}$ and $H_{(n)}^{R}$ for $n \geqslant 1$ we only need $\Phi_{(m)}^{l}, m<n$, and $n=0$ is the local case.

The methods developed in this section can also be applied to systems with infinitely many degrees of freedom, i.e., field theories.

We can consider, for instance, the Lagrangian

$$
L(t)=\int d^{3} \mathbf{x} \mathscr{E}(t, \mathbf{x} ;[\phi])
$$

with Lagrangian density

$$
\mathscr{L}=-\frac{1}{2} \phi_{\mu} \phi^{\mu}-\frac{1}{2} m^{2} \phi^{2}+\frac{g}{3 !} \tilde{\phi}^{3},
$$

where

$$
\tilde{\phi}=\frac{i e^{a m^{2}}}{(2 \sqrt{a \pi})^{4}} \int d^{4} y e^{y^{\mu} y_{\mu} / 4 a} \phi(y+x)=e^{a\left(\square+m^{2}\right)} \phi(x),
$$

which is relevant in string field theory. ${ }^{4}$

The symplectic form we obtain is

$$
\begin{aligned}
& \Omega^{R}=\Omega_{0}^{R}+g \Omega_{1}^{R}+O\left(g^{2}\right), \quad \Omega_{0}^{R}=\int d^{3} \mathbf{p} \delta \dot{\phi}(\mathbf{p}, t) \wedge \delta \phi(\mathbf{p}, t), \\
& \Omega_{1}^{R}=(2 \pi)^{-3 / 2} \int d^{3} \mathbf{p} d^{3} \mathbf{p}^{\prime} \sum_{A, B, C= \pm} S^{A B C}\left(\mathbf{p}, \mathbf{p}^{\prime}\right) a_{A}(\mathbf{p}, t) \delta a_{B}\left(\mathbf{p}^{\prime}, t\right) \wedge \delta a_{C}\left(\mathbf{p}+\mathbf{p}^{\prime}, t\right),
\end{aligned}
$$

and the Hamiltonian

$$
\begin{gathered}
H^{R}=H_{0}^{R}+g H_{1}^{R}+O\left(g^{2}\right) \\
H_{0}^{R}=\int d^{3} \mathbf{p}\left[\frac{1}{2} \dot{\phi}(\mathbf{p}, t) \dot{\phi}(-\mathbf{p}, t)+\frac{1}{2}\left(\mathbf{p}^{2}+m^{2}\right) \phi(\mathbf{p}, t) \phi(-\mathbf{p}, t)\right], \\
H_{1}^{R}=(2 \pi)^{-3 / 2} \int d^{3} \mathbf{p} d^{3} \mathbf{p}^{\prime} \sum_{A B C= \pm} \frac{i C \omega_{p+p^{\prime}}}{2} S^{A B C}\left(\mathbf{p}, \mathbf{p}^{\prime}\right) a_{A}(\mathbf{p}, t) a_{B}\left(\mathbf{p}^{\prime}, t\right) a_{C}\left(\mathbf{p}+\mathbf{p}^{\prime}, t\right),
\end{gathered}
$$


where

$$
S^{A B C}\left(\mathbf{p}, \mathbf{p}^{\prime}\right)=\mathscr{P} \frac{i e^{a\left(\omega_{p+p^{\prime}}^{2}-\left(A \omega_{p}+B \omega_{p^{\prime}}\right)^{2}\right)}}{A \omega_{p}+B \omega_{p^{\prime}}+C \omega_{p+p^{\prime}}}
$$

and

$$
\omega_{p} \equiv \sqrt{\mathbf{p}^{2}+m^{2}}
$$

As before, the elementary Poisson brackets are

$$
\begin{aligned}
\left\{\phi(\mathbf{p}), \phi\left(\mathbf{p}^{\prime}\right)\right\}= & \frac{g(2 \pi)^{-3 / 2}}{2 \omega_{p-p^{\prime}}} \\
& \times \sum_{B= \pm}\left[\mathscr{P}_{B \omega_{p} \kappa^{B}\left(p, p^{\prime}\right)}^{e^{-a \kappa^{B}\left(p, p^{\prime}\right)}} \dot{\phi}\left(\mathbf{p}^{\prime}-\mathbf{p}, t\right)-\mathscr{P}_{B \omega_{p^{\prime}} \kappa^{B}\left(p^{\prime}, p\right)} e^{-a \kappa^{B}\left(p^{\prime}, p\right)} \dot{\phi}\left(\mathbf{p}-\mathbf{p}^{\prime}, t\right)\right]
\end{aligned}
$$

with

$$
\kappa^{ \pm}\left(p, p^{\prime}\right)=\left(\omega_{p-p^{\prime}} \pm \omega_{p}\right)^{2}-\omega_{p^{\prime}}^{2}
$$

In order to compare with the results given in Ref. 4 we can consider the same system in terms of light cone coordinates $\left(x^{+}, x^{-}, x^{1}, x^{2}\right)$, with

$$
x^{ \pm}=\frac{1}{\sqrt{2}}\left(x^{0} \pm x^{3}\right)
$$

The zeroth order perturbative equation is then

$$
2 i p^{+} \dot{\phi}\left(x^{+},-\mathbf{p},-p^{+}\right)-\left(\mathbf{p}^{2}+m^{2}\right) \phi\left(x^{+},-\mathbf{p},-p^{+}\right)=0 .
$$

Taking $x^{+}$as evolution parameters, $\mathbf{p}=\left(p^{1}, p^{2}\right)$ and $p^{+}=1 / \sqrt{2}\left(p^{1}+p^{3}\right)$ as canonical momenta, and defining $\omega_{p}=\left(\mathbf{p}^{2}+m^{2}\right) / 2 p^{+}$, we arrive at the symplectic form

$$
\begin{gathered}
\Omega_{0}^{R}=\int d p^{+} d^{2} \mathbf{p}-i p^{+} \delta \phi\left(x^{+},-\mathbf{p},-p^{+}\right) \wedge \delta \phi\left(x^{+}, \mathbf{p}, p^{+}\right) \\
\Omega_{1}^{R}=-i(2 \pi)^{-3 / 2} \int d p^{+} d^{2} \mathbf{p} d p^{\prime+} d^{2} \mathbf{p}^{\prime} d p^{\prime \prime} d^{2} \mathbf{p}^{\prime \prime} \delta\left(p^{+}+p^{\prime+}+p^{\prime+}\right) \delta^{2}\left(\mathbf{p}+\mathbf{p}^{\prime}+\mathbf{p}^{\prime \prime}\right) \\
\times S\left(p, p^{\prime}, p^{\prime \prime}\right) \phi\left(x^{+}, \mathbf{p}^{\prime}, p^{\prime+}\right) \delta \phi\left(x^{+}, \mathbf{p}^{\prime \prime}, p^{\prime+}\right) \wedge \delta \phi\left(x^{+}, \mathbf{p}, p^{+}\right)
\end{gathered}
$$

and the Hamiltonian

$$
\begin{gathered}
H_{0}^{R}=\int d p^{+} d^{2} \mathbf{p} \frac{1}{2}\left(\mathbf{p}^{2}+m^{2}\right) \phi\left(x^{+},-\mathbf{p},-p^{+}\right) \phi\left(x^{+}, \mathbf{p}, p^{+}\right) \\
H_{1}^{R}=\frac{(2 \pi)^{-3 / 2}}{2} \int d p^{+} d^{2} \mathbf{p} d p^{\prime+} d^{2} \mathbf{p}^{\prime} d p^{\prime \prime} d^{2} \mathbf{p}^{\prime \prime} \delta\left(p^{+}+p^{\prime+}+p^{\prime+}\right) \delta^{2}\left(\mathbf{p}+\mathbf{p}^{\prime}+\mathbf{p}^{\prime \prime}\right) \\
\times \omega_{p} S\left(p, p^{\prime}, p^{\prime \prime}\right) \phi\left(x^{+}, \mathbf{p}, p^{+}\right) \phi\left(x^{+}, \mathbf{p}^{\prime}, p^{\prime+}\right) \phi\left(x^{+}, \mathbf{p}^{\prime \prime}, p^{\prime \prime}\right)
\end{gathered}
$$


with

$$
S\left(p, p^{\prime}, p^{\prime \prime}\right)=e^{3 a m^{2}} e^{-2 a\left(\omega_{p}+\omega_{p^{\prime}}+\omega_{p}{ }^{\prime \prime}\right) p^{+}} \mathscr{P} \frac{1}{\omega_{p}+\omega_{p^{\prime}}+\omega_{p}^{\prime \prime}}
$$

and the elementary Poisson brackets

$$
\begin{aligned}
\left\{\phi\left(x^{+}, p\right), \phi\left(x^{+}, p^{\prime}\right)\right\}= & \frac{i}{2 p^{+}} \delta\left(p^{+}+p^{\prime+}\right) \delta^{2}\left(\mathbf{p}+\mathbf{p}^{\prime}\right) \\
& +g(2 \pi)^{-3 / 2} \int d p^{\prime \prime} d^{2} \mathbf{p}^{\prime \prime} \delta\left(p^{+}+p^{\prime+}+p^{\prime+}\right) \delta^{2}\left(\mathbf{p}+\mathbf{p}^{\prime}+\mathbf{p}^{\prime \prime}\right) \\
& \times A\left(p, p^{\prime}, p^{\prime \prime}\right) \phi\left(x^{+},-\mathbf{p}^{\prime \prime},-p^{\prime+}\right),
\end{aligned}
$$

where

$$
A\left(p, p^{\prime}, p^{\prime \prime}\right)=\frac{i e^{3 a m^{2}}}{4 p^{+} p^{\prime+}}\left[e^{-2 a\left(\omega_{p}+\omega_{p^{\prime}}+\omega_{p}^{\prime \prime}\right) p^{+}}-e^{-2 a\left(\omega_{p}+\omega_{p^{\prime}}+\omega_{p}^{\prime \prime}\right) p^{\prime+}}\right] \mathscr{P} \frac{1}{\omega_{p}+\omega_{p^{\prime}}+\omega_{p^{\prime \prime}}},
$$

which writing $a=1 / m^{2}$ yields the same result as given in Eq. (3.104) in Ref. 4 .

\section{CONCLUSION AND OUTLOOK}

The construction of a Hamiltonian formalism for nonlocal Lagrangian systems has been accomplished in two steps. The first consisted in establishing a presymplectic formalism on the phase space of the nonlocal Lagrangian system. This is not yet a Hamiltonian formalism, because we are not able to prove, for the general case, that the presymplectic form so obtained is actually symplectic (i.e., regular), thus having an associated Poisson bracket structure. The main difficulty in proving this comes from the fact that a minimal set of independent data (either finite or infinite) parametrizing each solution of the Euler-Lagrange equations is not well determined in general.

The second step consisted in restricting ourselves to systems whose phase space has finitely many dimensions, either by itself (e.g., local Lagrangians) or by what is called order reduction. We have seen how local, regular Lagrangians can be included in our framework, and proven that our methods yield the well known Hamiltonian formalism that is obtained in the standard ways.

We have also considered the case of nonlocal "derived" Lagrangians, whose phase space also has finitely many dimensions. In this case our method is simpler to apply and clearly less artificial than the approaches based on Lagrangians that depend on the derivatives of any order.

Genuine nonlocal Lagrangians are not so easy to deal with. However, most nonlocal Lagrangians occurring in theoretical physics can be written as: $L=L_{0}+g L_{1}$ where $L_{0}$ corresponds to the free part and is a local first order Lagrangian, and all the nonlocal contribution comes from $L_{1}$. The Euler-Lagrange equations for $L$ are of functional differential type, and the phase space has infinitely many dimensions. It might be, however, that resorting to physical reasons, only perturbative solutions, namely, solutions that admit a formal expansion in powers of $g$, were interesting from a physical viewpoint. We have shown, with two examples, that the space of perturbative solutions can be parametrized by a finite number of coordinates (actually, in the same number as the nonperturbed system, $L_{0}$. We have also seen (Sec. VI C) that, if $L_{0}$ is regular, the presymplectic structure defined in the first part of the work, when restricted to the phase subspace of perturbative solutions, yields a symplectic structure and a Poisson bracket formalism.

There are two points concerning the power of tools here developed that are worth stressing. First, the process of construction of presymplectic formalism is thoroughly independent of any perturbative expansion, unlike the case in other previous attempts. And second, we have advanced a set of formulas, namcly, (54) and (55). The application of these formulas to any given nonlocal Lagrangian only requires integration of the nonperturbed system [Eq. (83)], the integration of a 
linear ordinary differential system [Eq. (85)], and the calculation of some integrals. Although these operations can be sometimes cumbersome, they merely involve mechanical manipulations.

Not surprisingly, it usually happens that the final elementary Poisson brackets $\left\{q_{i}, q_{j}\right\}$ do not vanish, but are first order with respect to $g$ (this is a familiar result in the relativistic theory of direct interaction, where the no interaction theorems apply). ${ }^{21}$ Hence, the coordinates of the original configuration space are not canonical coordinates. One way of continuing the present work would be the determination of possible canonical coordinates and momenta, and the subsequent canonical quantization.

\section{ACKNOWLEDGMENTS}

This work has been supported by DIGICyT, Contracts No. PB90-0012 and PB90-0482 and by the Societat Catalana de Fisica (Institut d'Estudis Catalans).

\section{APPENDIX: PROOF OF SUMMABILITY CONDITIONS}

We shall now show that the first and second terms on the right hand side of Eq. (28) fulfill the summability conditions (22), provided that $\zeta^{l}$ is a solution of the Euler-Lagrange Eqs. (4).

For the first term we must prove that

$$
\mathscr{J} \equiv \int_{\mathrm{R}}^{2} d \lambda d \sigma \chi(\lambda, \sigma) E_{l}(t-\sigma, t+\lambda ;[q]) \varphi(\lambda)<\infty
$$

for any slowly increasing [see condition (9)] continuous function $\varphi(\lambda)$.

Defining $\mathscr{\mathscr { D }}_{I} \equiv\{\lambda>0, \sigma>0\} ; \mathscr{D}_{I I} \equiv\{\lambda<0, \sigma<0\}$ we can write

$$
|\mathscr{J}| \leqslant\left(\int_{\mathscr{V}_{I}}+\int_{\mathscr{S}_{I I}}\left|E_{l}(t-\sigma, t+\lambda ;[q])\right||\varphi(\lambda)|\right.
$$

and, since $\mathscr{D}_{I I}$ transforms with $\mathscr{D}_{I}$ upon replacement of $(\lambda, \sigma)$ by $(-\lambda,-\sigma)$, it will be enough to prove the convergence of the integral over $\mathscr{D}_{l}$. (The proof for $\mathscr{D}_{I I}$ is quite similar.)

According to (7), there exists $L_{1}>0$ such that

$$
\left|E_{l}(t-\sigma, t+\lambda)\right|<\frac{1}{N(\lambda+\sigma)}
$$

whenever $\lambda+\sigma \geqslant L_{1}$.

Hence, the integral over $\mathscr{Q}_{l}$ in (115) has the upper bound

$$
\begin{aligned}
\int_{\mathscr{V}_{I}} \leqslant \int_{\lambda>0 ; \sigma>0 ; \lambda+\sigma \leqslant L} d \lambda d \sigma|E(t-\sigma, t+\lambda)||\varphi(\lambda)| \\
\quad+\int_{\lambda>0 ; \sigma>0 ; \lambda+\sigma>L} d \lambda d \sigma \frac{\Phi(\lambda+\sigma)}{N(\lambda+\sigma)} \forall L \geqslant L_{1},
\end{aligned}
$$

where $\Phi(\lambda)$ is the increasing, continuous, positive function defined by

$$
\Phi(\lambda) \equiv \max \left\{\left|\varphi\left(\lambda^{\prime}\right)\right| ; 0 \leqslant \lambda^{\prime} \leqslant \lambda\right\} .
$$

It is immediate to see that the condition (9) also holds replacing $|\varphi(\lambda)|$ by $\Phi(\lambda)$. Hence, there exists $L_{2}>0$ such that

$$
\Phi(\lambda)<N(\lambda) \cdot \lambda^{-\alpha} \quad(\alpha>2)
$$


whenever $\lambda>L^{2}$.

And using (A4) in (A3), we find that

$$
\int_{L_{I}} \leqslant \int_{\substack{\lambda>0 ; \sigma>0 \\ \lambda+\sigma \leqslant L}} d \lambda d \sigma|E(t-\sigma, t+\lambda)||\varphi(\lambda)|+\int_{\substack{\lambda>0 ; \sigma>0 \\ \lambda+\sigma>L}} d \lambda d \sigma(\lambda+\sigma)^{-\alpha} L \geqslant \max \left\{L_{1}, L_{2}\right\}
$$

or

$$
\int_{\mathscr{Q}_{1}} \leqslant \int_{\substack{\lambda>0 ; \sigma>0 \\ \lambda+\sigma \leqslant L}} d \lambda d \sigma|E(t-\sigma, t+\lambda)||\varphi(\lambda)|+\int_{L}^{\infty} d \mu \mu^{-\alpha+1}
$$

and both terms in the right hand side converge for $\alpha>2$. Hence, $\int_{\mathscr{C}_{I}}<+\infty$.

Since $\mathscr{Q}_{I I}$ converts into $\mathscr{D}_{I}$ upon replacement of $(\lambda, \sigma)$ by $(-\lambda,-\sigma)$, we can similarly prove that $\int_{\mathscr{C}}<+\infty$.

Hence $\| \mathscr{J} \mid \leqslant \int_{\mathscr{Q}_{1}}+\int_{\mathscr{Q}_{l l}}<+\infty$.

Q.E.D.

As for the second term in Eq. (28), we must prove that

$$
\int d \lambda K_{l}(t+\lambda) \varphi(\lambda)<\infty
$$

But this follows immediately from the facts that

$$
\int d \lambda K_{l}(\lambda) \varphi(\lambda-t)
$$

that the slow growth condition (9) holds for $\varphi(\lambda-t)$ provided that it does for $\varphi(\lambda)$, and that $K_{l}(\lambda)$ itself meets the summability condition $(10)$.

'A. D. Fokker, Z. Phys. 58, 386 (1929); J. A. Wheeler and R. P. Feynman, Rev. Mod. Phys. 21, 425 (1945).

${ }^{2}$ A. Pais and G. E. Uhlenbeck, Phys. Rev. 79, 145 (1950); R. Marnclius, Phys. Rev. D 8, 2472 (1973); D. Evens, J. W. Moffat, G. Kleppe, and R. P. Woodard, Nonlocal Regularizations of Gauge Theories, UTPT-90-08, UFIFT-HEP-90-15 (1990).

${ }^{3}$ P. Kristensen and C. Moller, K. Dan. Vidensk. Selsk. Matt-Fys. Medd. 27, 7 (1952); P. Havas, Phys. Rev. 86, 974 (1952).

${ }^{4}$ D. A. Eliezer and R. P. Woodard, Nucl. Phys. B 325, 389 (1989).

${ }^{5}$ H. Hata, Phys. Lett. B 217, 438 (1989).

6J. Z. Simon, Phys. Rev. D 43499 (1991).

${ }^{7}$ V. Arnold, Les Méthodes Mathématiques de la Mécanique Classique (MIR, Moscow, 1976), p. 202.

${ }^{8}$ L. Pontriaguine, Équations Différentielles Ordinaires (MIR, Moscow, 1975), p. 23.

${ }^{9} \mathrm{C}$. Crnkovic, Symplectic geometry of the covariant phase space, superstrings and superspace, PUPT-1059 (1987).

${ }^{10}$ E. J. Kerner, J. Math. Phys. 3, 35 (1962).

${ }^{11}$ R. Marnelius, Phys. Rev. D 102335 (1974).

${ }^{12}$ R. P. Gaida and V. I. Tretyak, Acta Phys. Pol. B 11, 509 (1980).

${ }^{13} \mathrm{E}$. Whittaker, A Treatise on the Analytical Dynamics of Particles and Rigid Bodies (Cambridge University, Cambridge, England, 1970).

${ }^{14}$ X. Jaén, J. Llosa, and A. Molina, Phys. Rev. D 34, 2302 (1986).

${ }^{15}$ X. Jaén, R. Jáuregui, J. Llosa, and A. Molina, Phys. Rev. D 36, 2385 (1987).

${ }^{16}$ L. Bel, Relativistic Action at a Distance: Classical and Quantum Aspects, Lecture Notes in Physics, Vol. 162 , p. 26 (Springer-Verlag, Berlin, 1982).

${ }^{17}$ A similar trick involving Lagrange multipliers, applied in the context of Lagrangians that depend on higher derivatives up to a finite order, can be found in P. W. Hebda, J. Math. Phys. 31, 2116 (1990), and R. Arens, Pacific J. Math. 93, 1 (1981).

${ }^{18}$ Y. Choquet-Bruhat, C. DeWitt-Morette, and M. Dillard-Bleick, Analysis, Manifolds and Physics (North-Holland, Amsterdam, 1987), Sec. III-C.1.

${ }^{19}$ Reference 18 , Sec. IV-C. 10.

${ }^{20}$ Reference $18 \mathrm{Sec}$. III-B.4.

${ }^{21} \mathrm{~L}$. Bel and H. Sirousse Zia, Phys. Rev. D 32, 3128 (1985).

${ }^{22}$ R. E. O'Malley, Introduction to Singular Perturbations (Academic, New York, 1974).

${ }^{23}$ D. G. Currie, T. F. Jordan, and E. C. G. Sudarshan, Rev. Mod. Phys. 35, 350 (1963). 